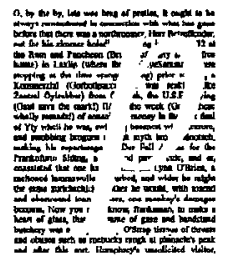

\title{
Configuraciones descriptivas: articulaciones simbólicas e ideológicas en la narrativa de ficción
}

\author{
Luz Aurora Pimentel
}

\section{Universidad Nacional Autónoma de México}

$\mathbf{U}$ na descripción es un fenómeno de expansión textual que consiste en hacer equivaler una nomenclatura y una serie predicativa. La nomenclatura, $o$ tema descriptivo propuesto, generalmente éstá constituida, o bien por nombres propios con una fuerte orientación referencial, o bien por lexemas nominales cuya organización semántica interna determina, potencialmente, la forma y dirección de ese despliegue sintagmático que es la descripción. Describir una casa, por ejemplo, es enumerar, con mayor o menor detalle, sus habitaciones, puertas, ventanas, techos, muros ... etc. En general, el nombre del objeto a describir tiende a enunciarse primero - en una suerte de incipit de la descripción—luego se despliega en una serie de atributos, partes yło «detalles.» En la base de la serie predicativa está la organización semántica interna del lexema propuesto como tema descriptivo. De ahí que la enumeración, el catálogo o el inventario constituya la forma de organización básica de una descripción. Sin embargo, esa serie es, en principio, ilimitada; se pueden ofrecer uno o mil detalles para construir la «imagen» de un lugar, de un objeto o de una persona; no hay nada inherente al tema descriptivo que marque el principio o el fin de la serie. Es por ello que el narrador-descriptor se ve obligado a poner coto al inventario sin fin, que de otro modo podría ser una descripción, con la ayuda de modelos de organización suplementarios que den la ilusión de que los límites son inherentes al objeto descrito y no a la forma de organización elegida. Los modelos pueden ser de tipo lógico-lingüistico como el de las dimensiones (adentro, afuera; encima, debajo; arriba, abajo, al centro, al fondo; a la izquier- 
da, a la derecha, etc.); modelos de tipo taxonómico (las distintas partes de un árbol, de una planta, del cuerpo humano, etc.), temporal (las horas del día, lás estaciones del año, etc.), o cultural (el modelo de la pintura que permite describir un lugar como si fuera un cuadro; modelos arquitectónicos, musicales, entre otros). Saturar el o los modelos elegidos contribuye a ordenar la descripción pero también a crear la impresión de que el final de la descripción es «natural» y «necesario.» Más aún, el modelo utilizado para organizar una descripción le da una unidad temática que implica su continuidad semántica. incluso si aparece de manera fragmentaria en la organización textual de un' relato. ${ }^{1}$

Ahora bien, la forma paratáctica de la enumeración, como rasgo distintivo de la descripción, tiende a aparecer incluso en organizaciones textuales más complejas. Estas últimas generalmente están determinadas, como hemos visto, por los modelos propuestos por otros discursos, y a ellos se subordina el desarrollo descriptivo. La serie predicativa, organizada por modelos extratextuales, establece en su interior una serie de jerarquizaciones internas informadas por un doble movimiento: el uno hacia lo general (la visión de conjunto); hacia lo particular el otro (los detalles).

De este modo, la descripción como un todo, ordenado de acuerdo ćon el esquema propuesto por un modelo pre-existente, se nos presenta como un sistema, dentro del cual todas sus partes constitutivas se interrelacionan para constituirse en un todo significante. No obstante, independientemente de la organización propuesta por el modelo elegido, al interior mismo de un sistema descriptivo con frecuencia aparecen ciertas particularidades del objeto descrito en la forma de un conjunto ordenado, más o menos autónomo. Se trata de un arreglo de semas o partes, local y particularr, más allá del modelo general que organiza la descripción como un todo; una disposición de rasgos semánticos que produce una especie de «figura,» y que no se reconoce como tal mientras no se repita en algún otro punto del texto. Llamaremos a este tipo de ordenamiento local una configuración descriptiva: ${ }^{2}$ ciertas partes de la serie predicativa, al ser descritas, se ordenan de un modo particular, o guardan relaciones especiales que generan una cierta significación, y que, más tarde, habiendo abstraído de ese arreglo particular un patrón semántico, el patrón o configuración ha de reduplicarse en algún otro sistema descriptivo. Dicho de otro modo, y atendiendo al medio narrativo en el que operan estas configuraciones descriptivas, puede decirse que en el curso de la lectura de un texto narrativo, el lector percibe en una secuencia descriptiva el modo en que están dispuestas ciertaș partes o detalles de la secuencia. Más tarde, en la descripción de algún otro objeto, reconoce el mismo arreglo semántico, a pesar de la diferencia en los objetos descritos. Al abstraer de la diversidad lingüística y temática los mismos rasgos semánticos ordenados e interrelacionados de la misma manera, se construye un 
da, a la derecha, etc.); modelos de tipo taxonómico (las distintas partes de un árbol, de una planta, del cuerpo humano, etc.), temporal (las horas del día, las estaciones del año, etc.), o cultural (el modelo de la pintura que permite describir un lugar como si fuera un cuadro; modelos arquitectónicos, musicales, entre otros). Saturar el o los modelos elegidos contribuye a ordenar la descripción pero también a crear la impresión de que el final de la descripción es "natural» y «necesario.» Más aún, el modelo utilizado para organizar una descripción le da una unidad temática que implica su continuidad semántica incluso si aparece de manera fragmentaria en la organización textual de un relato.'

Ahora bien, la forma paratáctica de la enumeración, como rasgo distintivo de la descripción, tiende a aparecer incluso en organizaciones textuales más complejas. Estas últimas generalmente están determinadas, como hemos visto, por los modelos propuestos por otros discursos, y a ellos se subordina el desarrollo descriptivo. La serie predicativa, organizada por modelos extratextuales, establece en su interior una serie de jerarquizaciones internas informadas por un doble movimiento: el uno hacia lo general (la visión de conjunto); hacia lo particular el otro (los detalles).

De esté modo, la descripción como un todo, ordenado de acuerdo con el esquema propuesto por un modelo pre-existente, se nos presenta como un sistema, dentro del cual todas sus partes constitutivas se interrelacionan para constituirse en un todo significante. No obstante, independientemente de la organización propuesta por el modelo elegido, al interior mismo de un sistema descriptivo con frecuencia aparecen ciertas particularidades del objeto descrito en la forma de un conjunto ordenado, más o menos autónomo. Se trata de un arreglo de semas o partes, local y particular, más allá del modelo general que organiza la descripción como un todo; una disposición de rasgos semánticos que produce una especie de «figura,» y que no se reconoce como tal mientras no se repita en algún otro punto del texto. Llamaremos a este tipo de ordenamiento local una configuración descriptiva: ${ }^{2}$ ciertas partes de la serie predicativa, al ser descritas, se ordenan de un modo particular, o guardan relaciones especiales que generan una cierta significación, y que, más tarde, habiendo abstraído de ese arreglo particular un patrón semántico, el patrón o configuración ha de reduplicarse en algún otro sistema descriptivo. Dicho de otro modo, y atendiendo al medio narrativo en el que operan estas configuraciones descriptivas, puede decirse que en el curso de la lectura de un texto narrativo, el lector percibe en una secuencia descriptiva el modo en que están dispuestas ciertas partes o detalles de la secuencia. Más tarde, en la descripción de algún otro objeto, reconoce el mismo arreglo semántico, a pesar de la diferencia en los objetos descritos. Al abstraer de la diversidad lingüística y temática los mismos rasgos semánticos ordenados e interrelacionados de la misma manera, se construye un 
patrón semántico abstracto que subyace y conecta secuencias descriptivas textualmente discontinuas, organizadas en torno a temas descriptivos diferentes. Estas conexiones son generadoras de importantes significados narrativos que, con frecuencia, permiten las más variadas articulaciones ideológicas y simbólicas en un texto narrativo.

En la base de esta construcción de lectura está la repetición, pues es solamente en el momento en que se repite un mismo arreglo semántico que se instituye en configuración reconocible. Más aún, habría que insistir en que, por una parte, la redundancia es de orden semántico y no puramente léxico; por otra, que este conjunto ordenado de semas o partes no es sinónimo de, ni coincide con, la constitución semántica o morfológica del objeto descrito; el conjunto está constituido por algunos de los semas o partes constitutivas y por semas de orden connotativo o aferente, ordenados todos de tal manera que formen un patrón o una figura semántica abstracta que más tarde pueda reconocerse en otra descripción; es decir, en la descripción de otro objeto diferente.

Es importante subrayar que la configuración descriptiva es una construcción abstraida de las variaciones que puedan darse en el nivel de la manifestación lingüística o en el del contenido de la descripción. Esto quiere decir que objetos diferentes pueden describirse utilizando una misma configuración, lo cual puede establecer entre ambas descripciones relaciones significantes de diversos tipos. En la base de estas configuraciones está la iteratividad, ya que si no se reduplicara la figura dibujada por una descripción, no sería posible aislar estas partes de la serie predicativa en un subconjunto, ordenado de manera particular en configuración descriptiva. Examinemos ahora un par de descripciones provenientes de Naná, de Emile Zolá, con objeto de ilustrar algunos de estos procesos de articulación simbólica.

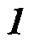

Le jour enfin se leva, ce petit jour sale des nuits d'hiver, si mélancholique sur le pavé boueux de Paris. ${ }^{3}$

Por fin amaneció, con ese sucio crepúsculo de las noches de invierno, tan melancólico sobre el cenagoso empedrado de Paris. ${ }^{4}$

2

Alors, c'était avec Satin des sorties enragées sur le pavé de Paris, dans ce vice d'en bas qui rôde le long des ruelles boueuses, sous la clarté trouble du gaz. (273)

Entonces, en compañia de Satin, emprendía obstinadas carreras sobre el empedrado de París, en ese vicio bajo que ronda por las callejuelas cenagosas, a la turbia claridad del gas. (142) 
Les deux femmes se lancèrent dans leur souvenirs. Ça les prenait par crises bavardes; elles avaient une brusque besoin de remuer cette boue de leur jeunesse; et c'était toujours quand il y avait là des hommes, comme si elles cédaient à une rage de leur imposer le fumier où elles avaient grandi. (335)

Las dos jóvenes se engolfaron en sus recuerdos. Eran como crisis parlanchinas; sentian una brusca necesidad de remover aquel lodo de los albores de su juventud, y siempre ocurría cuando habia alli hombres, como si cediesen a un frenesi de imponerles el estercolero en que habian crecido. (177)

\section{$\boldsymbol{B}$}

L'avenue de Villiers, déserte, allongeait la double file de ses becs à gaz, au fond de cette nuit humide de mars ... Des terrains vagues faisaient des trous de ténèbres; des hôtels en construction dressaient leurs échafaudages sous le ciel noir ...

Satin s'était serrée contre Nana, dans un petit frisson. Pourtant, elles restèrent, intéressées par l'approche d'une lanterne, dansante au milieu des flaques'de la chaussée. C'était une vieille chiffonnière qui fouillait les ruisseaux. Satin la reconnu ...

Et, tandis qu'un coup de vent leur fouettait à la face une poussière d'eau, elle racontait à sa chérie l'histoire de la reine Pomaré ... Enfin une vraie dégringolade, une reine tombée dans la crotte! ...

La chiffonnière, qui se trouvait sous la fenêtre, leva la tête et se montra, à la lueur jaune de sa lanterne. C'était, dans ce paquet d'haillons, sous un foulard en loques, une face bleuie, couturée, avec le trou édenté de la bouche et les meurtrissures enflammées des yeux. (345)

La avenida de Villiers, desierta, extendía la doble fila de sus mecheros de gas en el fondo de aquella húmeda noche de marzo ... Los terrenos sin edificar formaban agujeros en las tinieblas; los hoteles en construcción erguian sus andamiajes bajo el negro cielo ...

Satin se apretaba contra Naná, estremeciéndose ligeramente. Sin embargo, no se apartaron de la ventana, interesadas por la aproximación de una linterna danzante en medio de los charcos de la calzada. Era una vieja trapera que escudriñaba los arroyos. Satin la reconoció... refirió a su amiga la historia de la reina Pomaré... En resumen; una verdadera voltereta; una reina caída en el fango ...

La trapera, que se hallaba al pie de la ventana, levantó la cabeza, y se mostró al amarillento resplandor de la linterna.

Entre aquel paquete de andrajos, bajo un pañuelo hecho jirones, vióse un rostro amoratado, lleno de arrugas, con el agujero desdentado de la boca, $y$ las orejas lividas inflamadas. (182-83) 


\section{C}

Nana restait seulé, la face en l'air, dans la clarté de la bougie. C'était un charnier, un tas d'humeur et de sang, une pelletée de chair corrompue, jetée là, sur un cousin. Les pustules avaient envahi la figure entière, un bouton touchant l'autre; et, flétries, affaissées, d'un aspect grisâtre de boue, elles semblaient déjà une moisssure de la terre, sur cette bouillie informe, où l'on ne retrouvait plus de traits. Un oeil, celui de gauche, avait complètement sombré dans un bouillonnement de la purulence; l'autre, à demi ouvert, s'enfonçait, comme un trou noir et gâté. Le nez suppurait encore. Toute une croûte rougeâtre partait d'une joue, envahissait la bouche, qu'elle tirait dans un rire abominable. Et, sur ce masque horrible et grotesque du néant, les cheveux, les beaux cheveux, gardant leur flambée de soleil, coulaient en un ruissellement d'or. Vénus se décomposait. (474)

Naná quedaba sola, boca arriba, a la claridad de la bujia. Era un osario, un montón de humores y de sangre, una paletada de carne putrefacta, arrojada alli sobre un colchón. Las pústulas habian invadido toda la cara, tocándose unas con otras; $y$ marchitas, hundidas, con su agrisado aspecto de lodo, parecian ya un enmohecimiento de la tierra sobre aquella papilla informe, donde ya no existian rasgos. Un ojo, el izquierdo, había desaparecido completamente en el hervor de la purulencia; el otro medio abierto, se hundia como un agujero negro y corrompido ... Y sobre aquella máscara horrible y grotesca de la nada, los cabellos, los hermosos cabellos, conservando sus reflejos del sol, corrian como chorros de oro. Venus se descomponía. (250)

En el bloque $A$, los pasajes seleccionados muestran un constante ir y venir, como reflejos especulares, entre el lodo de las calles de París y el lodo metafórico de las vidas de estas dos cortesanas. Este constante ir y venir del cieno urbano al moral acaba contaminando ambas realidades al grado de proponerlas como intercambiables: la calle y el lodo no sólo como el éntorno de la prostitución, sino como figuración de esa prostitución y del poder corruptor del dinero. Más aún, de manera muy sutil, el motivo de la luz comienza a insinuarse en esa insistente asociación entre el lodo, las calles y las prostitutas.

En el segundo pasaje, $B$, se reiteran los elementos que han quedado asociados en los pasajes anteriores. La descripción se inicia proyectando una calle en uno de los nuevos barrios de Paris. En un primer momento parecería tener sólo una función referencial inmediata: la avenida de Villiers es una representación realista de una calle que ostenta el mismo nombre en París y que constituye solamente el marco realista a esta parte del relato. Sin embargo, es interesante que esta descripción de la calle quede yuxtapuesta a la de una vieja mendiga. Es el principio mismo de la yuxtaposición textual lo que hace que la recurrencia de este patrón semántico sea perceptible: luz y oscuridad entreveradas en 
relación a un centro/hueco informe rodeado de luz, constituyen algunos de los elementos de esta configuración descriptiva.

Detengámonos un poco más en estos procedimientos descriptivos. Resalta en la descripción de la calle la insistencia en el contraste entre la luz y la oscuridad, figurado por la doble hilera de mecheros de gas alternando con los terrenos baldíos que se describen como "ragujeros en las tinieblas.» Surge de pronto la vieja trapera, de esos «agujeros») de tinieblas, como si la calle se hubiera personificado y la doble hilera de mecheros se hubiese animado en un entreverado de luz y oscuridad, una linterna danzante: «l'approche d'une lanterne, dansante au milieu des flaques de la chaussée.» La descripción se centra en su cara, que de alguna manera resume su historia como cortesana en decadencia, pero que también es un "eco»-por la forma en la que se la describe- de la calle recién descrita: el mismo contraste entre luz y oscuridad; la luz como una especie de marco sucio del rostro de la trapera y del borde de la calle; la oscuridad figurada en ambos casos como un «agujero»-interior o de alguna manera central, con respecto a la luz que lo enmarca-la doble hilera de mecheros de gas entreverados con los agujeros negros, informes («vagues») de los terrenos baldios y el agujero negro, igualmente informe, de la boca desdentada. Un patrón semántico reconocible se hà ido configurando de manera cumulativa al ser utilizado en distintos momentos a lo largo del relato. Podríamos ya, a estas alturas, resumir las distintas partes de esta configuración descriptiva: 1) luz y oscuridad entreveradas, o bien una zona de oscuridad rodeada por un halo de luz amarillenta; 2) agujeros, presentes en la calle, en los terrenos baldíos y en la boca desdentada de la trapera; 3) un centro informe; o bien formas vagas e indeterminadas: una significación contenida tanto en los terrenos baldíos («terrains vagues») como en los harapos de la vieja («paquet d'haillons») de entre los cuales emerge la cara de la vieja, como otro centro informe; $\mathrm{y}$, finalmente, 4) el lodo, en el que todos los demás elementos se mezclan de manera promiscua: el lodo está presente en las calles, en la vida de las prostitutas, y en la suciedad e indefinición de los harapos de la mendiga; más aún, el lodo también está presente, de manera insidiosa, en la luz, pues constantemente se la califica como turbia o sucia.

Vemos pues cómo esa configuración descriptiva recurrente, por las interrelaciones particulares que la definen, dibuja una figura abstracta que se repite en la descripción de dos objetos diversos: una calle y un rostro. De manera significativa, encontramos la misma figura nuevamente al final de la novela, en la extraoordinaria descripción del rostro desfigurado de Naná muerta (pasaje C): tanto la claridad de la bujía como los cabellos dorados, luminosos-y para ambos se subraya el matiz cromático del amarillo- enmarcan la carne informe y putrefacta en la que volvemos a encontrar un agujero negro, una carne 
convertida en cieno, «un emohecimiento de la tierra» («d'un aspect grisâtre de boue, elles semblaient déjà une moissure de la terre.») ${ }^{5}$

A lo largo de toda la novela, la belleza de Naná, y en especial su deslumbrante cabellera rubia, ha desplegado un abanico de significaciones que tienen un valor ideológico innegable. Como su fluida cabellera, el oro la cubre, corrompiéndolo todo; a su vez, el oro, emblemático de toda una red de relaciones morales, sociales y económicas, es devorado por una corrupción incontrolada e incontrolable. Oscuridad abismal, corrupción enmarcada por el oro y la luz: Naná y París figuran esa corrupción oculta tras el deslumbrante oropel de la aristocracia francesa y de las fabulosas fortunas despilfarradas (oro nuevamente) que acaban siendo devoradas por el agujero negro de la corrupción sexual. La fuerza del sentido articulado de esta manera reside no sólo en una denuncia programática, sino en la intensidad y complejidad de estas imágenes proyectadas en gran medida por ese patrón semántico abstracto que he denominado "configuración descriptiva.» Son esas configuraciones las que articulan la realidad ficcional, como universo de referencia en el que se inscribe el objeto descrito, y las significaciones del orden de lo ideológico y simbólico que ese mismo objeto descrito adquiere, gracias a la recurrencia del patrón semántico que la lectura ha construido. Es por eso que, como muchos estudiosos de la obra de Zola lo han observado, los universos ficcionales que crea el escritor naturalista tienen un alcance de significación tal que con mucho rebasan la declarada intención de veracidad científica, tan cara al proyecto naturalista, para incursionar en las densas zonas míticas de la realidad.

Las formas de articulación simbólico-ideológicas, a las que da pie una configuración descriptiva, pueden ser relativamente simples, incluso evidentes, como en los pasajes citados de Naná, o extremadamente complejos, como ocurre con frecuencia en el Ulises de James Joyce, por ejemplo. Examinemos un par de fragmentos tomados del episodio «Hades:» el primero describe el establecimiento de un constructor de monumentos fúnebres, ya casi para llegar al cementerio; el segundo, describe los monumentos fúnebres dentro del cementerio.

\section{$A$}

The stonecutter's yard on the right. Last lap. Crowded on the spit of land silent shapes appeared, white, sorrowful, holding out calm hands, knelt in grief, pointing. Fragments of shapes, hewn. In white silence: appealing. Thebest obtainable. Thos. H. Dennany, monumental builder and sculptor.

El corralón del picapedrero a la derecha. Último tramo. Apiñadas sobre la lengua de tierra aparecieron formas silenciosas, blancas, pesarosas, extendiendo calmas manos, arrodilladas en aflicción, señalando. Fragmentos de formas talladas en piedra. En blanco silencioso: suplicantes. Las mejores 
que se pueden conseguir. Thos. H. Dennany. Constructor de sepulcros $y$ escultor.

\section{$\boldsymbol{B}$}

The high railings of Prospects rippled past their gaze. Dark poplars, rare white forms. Forms more frequent, white shapes thronged amid the trees, white forms and fragments streaming by mutely, sustaining vain gestures on the air. ${ }^{6}$

Las altas verjas de Prospects ondearon bajo su mirada. Álamos oscuros, raras formas blancas. Formas más frecuentes, siluetas blancas apiñadas entre los árboles, blancas formas y fragmentos de monumentos pasando mudos, prolongados gestos vanos en el aire. ${ }^{7}$

En ambos fragmentos se describen monumentos fúnebres; lo que cambia es el entorno diegético (fuera y dentro del cementerio-rel corralón del picapedrero,» «las altas verjas de Prospects»); se altera también el ritmo descriptivo (relativamente estático en la primera descripción, dinámico en la segunda). Ahora bien, aun cuando en ambos el contenido de la descripción es parecido (tumbas y monumentos fúnebres), y a pesar de las diferencias rítmicas que tienen una importancia narrativa considerable, lo que llama la atención es la selección léxica, así como los detalles aislados que se focalizan. Es notable, por ejemplo, que en ningún momento se utilice la palabra «tombstone,» y que no se haga mención de los materiales de que están hechos los monumentos. ${ }^{8}$ Pero lo que la segunda descripción pone en evidencia es la relación significante que guardan todos estos elementos entre sí. Se hace hincapié en un cierto número de rasgos semánticos en ambas descripciones-independientemente de los lexemas que los expresen en el nivel de la manifestación-y la relación que guardan unos con otros, relación que se repite en la segunda descripción: 1)/tumulto/(manifiesto en los siguientes lexemas: «crowded,» «more frequent,» «thronged,» «streaming»); 2 )/forma/ (manifiesto en «shape» $\mathrm{y}$ «forms,» repetidos varias veces, en diversas combinaciones, en cada descripción); 3)/silencio/ (manifiesto en "pointing,» «silence,» repetido varias veces con distintas variantes, «mutely»); ${ }^{10}$ 4) /sufrimiento/ (manifiesto en «sorrowful,» «in grief,» "sustaining vain gestures on the air»); ${ }^{11}$ ) una significación afín a la anterior, la /súplica/ (manifiesta en «holding out calm hands,» «appealing,» «sustaining vain gestures on the air»); ${ }^{12} 6$ ) finalmente el color/blanco/asociado, de manera insistente, con las formas y fragmentos.

Son, pues, seis las unidades de significación que configuran estas dos descripciones: /tumulto/, /forma/, /blancura/, /silencio/, /sufrimiento/ y /súplical. En torno al tema descriptivo principal/formas/ - es decir, los «monumentos fúnebres»-las otras cinco unidades de significación las van particularizando. Se puede observar aquí un arreglo particular de los semas en torno 
al objeto de la descripción; empero, lo particular de esta organización sólo se percibe en el momento en que el arreglo se repite en la segunda descripción. De la redundancia es que surge la configuración descriptiva; al reduplicarse, la figura cristaliza en un patrón reconocible que le permite al lector proyectarla sobre otras figuras semánticas similares, ya sea de manera intratextual o intertextual, para construir formas de significación simbólica o ideológica. Así, la relación intratextual entre estos dos puntos del relato se funda en la recurrencia de una misma configuración descriptiva. Pero esa misma configuración descriptiva también es capaz de establecer relaciones intertextuales, como lo veremos a continuación.

Debido a la cristalización de la configuración descriptiva, con base en la redundancia, y debido a la invitación paratextual (Ulises) a establecer relaciones de analogía y/o de derivación con La Odisea, la configuración descriptiva examinada se convierte en el punto de articulación entre el texto de Joyce y el homérico, ya que en la sola configuración descriptiva quedan incorporados muchos de los temas y una buena parte del contenido narrativo del episodio correspondiente en La Odisea (Libro XI). ${ }^{13}$ Cuando Ulises llega al Hades, tras los ritos necesarios, aparecen los muertos como en enjambre; envueltos en el sufrimiento de la muerte, su actitud es suplicante (especialmente v. 84-96; 225-34).

Now when, with sacrifices and prayers, I had so entreated the hordes of the dead, I took the sheep and cut their throats over the pit, and the dark-clouding blood ran in, and the souls of the perished dead gathered to the place...

These came swarming around my pit from every direction. (v. 34-37; 42)

Después de haber rogado con votos y súplicas al pueblo de los difuntos, tomé las reses, las degollé encima del hoyo, corrió la negra sangre y al instante se congregaron, saliendo del Érebo, las almas de los fállecidos ... agitábanse todas con grandísimo murmurio alrededor del hoyo, unas por un lado y otras por otro.

These were all who had been the wives and daughters of princes, and now they gathered in swarms around the dark blood. (v. 227-8)

... cuantas mujeres fueron esposas o hijas de eximios varones. Reuniéronse en tropel alrededor de la negra sangre.

... there came the soul of Agamemnon, the son of Atreus, grieving, and the souls of other men, who died with him and met their doom in the house of Aigisthos, were gathered around him He knew me at once, when he drank the dark blood, and fell to lamentation loud and shrill, and the tears came springing. (v. 386-91)

... presentóse muy angustiada la de Agamenón Atrida; a cuyo alrededor se congregaban las de cuantos en la mansión de Egisto perecieron con el héroe, 
cumpliendo su destino. Reconocióme así que bebió la negra sangre y al punto comenzó a llorar ruidosamente: dẹramaba copiosas lágrimas.

The soul of swift-footed Achilleus, scion of Aiakos, knew me, and full of lamentation he spoke to me in winged words. (v. 471-2)

Reconocióme el alma del Eácida, el de los pies ligeros y lamentándose me dijo estas aladas palabras.

Now the rest of the souls of the perished dead stood near me grieving, and each one spoke to me and told of his sorrows. (v. 541-42)

Las otras almas de los muertos se quedaron aun y nos refirieron, muy tristes, sus respectivas cuitas.

(El subrayado es mío)

Queda implícito en este episodio de La Odisea el hecho de que a los muertos les ha sido vedado hablar - aun cuando "se agolpen en inhumano clamor»-a menos que Ulises les permita beber de la sangre ritual. De este modo la prohibición de hablar resuena en el insistente «silencio» de las figuras joyceanas. Como podrá observarse, una estrategia para el tejido intertextual reside no sólo en la transformación de lo narrativo en descriptivo, sino en la abstracción de la temporalidad de la acción para dibujar un patrón de significación puramente semántica. Dicho de otro modo, en este episodio de La Odisea están contenidos, en forma narrativa (y por tanto en una secuencia que temporaliza estos elementos), todos los significados que configuran la descripción correspondiente en el texto de Joyce, y que constituyen una forma metafórica de alusión al contenido narrativo del episodio correspondiente en el texto homérico, más que un intento de narrativización directa de esos contenidos temáticos. Lo que en Joyce es configuración abstracta en Homero es despliegue de acontecimientos referidos.

Es así como, para cada una de las seis unidades de significación que constituyen esta configuración descriptiva en Joyce, observamos un desarrollo narrativo en Homero. Dos de ellas, sin embargo, son menos explícitas- «forms» $\mathrm{y}$ «white»-debido a la operación de implicación que se necesita llevar a cabo para establecer una relación de analogía con el texto homérico: si en este último los muertos se nos presentan como espíritus descarnados que necesitan de la sangre para poder asumir forma y funciones humanas, en la descripción joyceana son los monumentos fúnebres (ese «tombstones» que nunca se menciona de manera explícita) los que, al describirlos, se han «descarnado,» por así decirlo, ya que el descriptor evade precisamente el nombre que les conferiría toda su materialidad - 《lápidas,» «monumentos fünebres,» etc. Es por eso que la no mención de los materiales de que están hechos los monumentos es una elección estética de capital importancia. Al evadir toda mención a los materiales, al referirse a los monumentos con un nombre más abstracto- «forms,» 
"shapes»-el objeto descrito pierde toda materialidad para entrar en relación metafórica con los pobladores del Hades. Por otra parte, éstos se describen en Homero como un pueblo que vive oculto en la bruma y las nubes, sin que Helios los ilumine jamás (v. 15-20):

There lie the community and city of Kimmerian people, hidden in fog and cloud, nor does Helios, the radiant sun, ever break through the dark, to illuminate them with his shining. (Book XI, v. 14-6)

Alli están el pueblo y la ciudad de los Cimerios entre nieblas y nubes, sin que jamás el sol resplandeciente los ilumine con sus rayos. (Libro XI, v. 14-1)

Si en el Hades griego este pueblo vive alejado del radiante Helios, sus pálidos correlatos metafóricos en Dublín padecen de la misma ausencia solar; de ahí que en el Hades joyceano las formas sean repetidamente «blancas.» Implícito en el color elegido está el complejo de significación aferente que pasa por la /palidez/como punto de articulación entre los dos términos fuertes: $/ \mathrm{sin}$ sol/, /sin sangre/.

Aun cuando la configuración descriptiva que hemos analizado establece relaciones que son de naturaleza metafórica, no puede decirse que la metáfora ocurra en la textura verbal de estas descripciones; es, de hecho, la recurrencia de un patrón semántico idéntico en la base de las diferencias contextuales y en los objetos descritos, lo que desencadena un proceso de articulación metafórica que desemboca en una dimensión paranarrativa, como construcción de lectura, que en otro momento he llamado narración metafórica. ${ }^{14}$ Ahora bien, el fenómeno de la configuraciọn descriptiva es también observable al interior de una metáfora, no ya como articulación narrativo descriptiva, sino presente en la textura verbał como figura de la retórica. Ofreceremos ahora una breve definición. Una metáfora es un fenómeno de confrontación, interacción y revaloración semánticas. Resulta de una predicación que violenta la coherencia semántica de un enunciado. A su vez, esa coherencia semántica, o isotopia, ${ }^{15}$ que establece el contexto principal de un enunciado es producto de una redundancia de semas o unidades mínimas de significado compatibles y del mismo grado de generalidad. En un verso como el de Rubén Darío, «Cisnes, los abanicos de vuestras alas frescas ... » la palabra «abanicos» es claramente incompatible con el contexto /animal/ que «cisnes» y «alas» establecen. En la interacción de los lexemas isotópicos («cisnes,» «alas») y alotópico («abanicos») se da una confrontación de dos grados de significación: el grado dado, manifiesto en la cadena sintagmática por la incompatibilidad de algunos lexemas con respecto a otros, y el grado construido, resultado de la interacción y revaloración de los dos campos semánticos en conflicto. ${ }^{16}$ Pero en la confrontación entre campos semánticos incompatibles, por vía de una interacción entre 
lexemas isotópicos y alotópicos, se observa una zona de coposesión de semas, o intersección sémica, ${ }^{17}$ que restablece—o mejor dicho, establece- el sentido metafórico del enunciado. Entre «alas» y "abanico,» una descomposición sémico-morfológica descubre una semejanza a partir de semas afines, tales como /(des)plegable/. Sobre la base de esta intersección se construye la significación metafórica como tal.

Ahora bien, con frecuencia la interacción metafórica se da no entre lexemas aislados («abanicos» y «alas,» -por ejemplo), ni siquiera entre grupos de lexemas, sino entre verdaderas configuraciones descriptivas. La intersección sémica, en estos casos, está constituida, no por la identidad de uno o varios semas aislados, sino por una configuración descriptiva idéntica tanto en el grado dado como en el construido de la metáfora. Acudiremos a Joyce nuevamente para ilustrar este fenómeno. En esta ocasión me referiré al episodio del periódico: «Eolo.» Al salir de las oficinas del periódico, Bloom es descrito desde la perspectiva de otros dos personajes que lo miran desde la ventana:

-... Look at the young scamps after him.

-Show. Where? Lenehan cried, running to the window ...

Both smiled over the crossblind at the file of capering newsboys in $\mathrm{Mr}$. Bloom's wake, the last zigzagging white on the breeze a mocking kite, ${ }^{18}$ a tail of white bowknots. (128)

-... Miren a esos pillos detrás de él.

- ¿Dónde? A ver ... - gritó Lenehan, corriendo a la ventana ... Ambos observaron sonriendo desde la persiana la fila de traviesos canallitas a la zaga del señor Bloom, el último de ellos haciendo zigzaguear blanco en la brisa un barrilete burlón, cola de nudos blancos. (160)

En esta descripción, se pueden leer dos metáforas simultáneamente, una menos explícita que la otra; $y$, por lo tanto, es posible aislar tres incidencias de la misma configuración descriptiva (una no metafórica, las otras dos metafóricas). En el nivel que pudiéramos llamar denotativo, o no metafórico de la descripción, se trata de un hombre caminando por la calle seguido de varios periodiqueros, con sus diarios bajo el brazo, saltando y haciendo piruetas en el aire («capering»), en son de burla. La descripción propone como metáfora explícita la del papalote («kite»). Si bien es cierto que la isotopía /aérea/, por así llamarla, se activa a partir de los lexemas «kite,» «tail of white bowknots» y «breeze,» la significación metafórica, empero, surge de la figura semántica que trazan los distintos elementos de la descripción y las relaciònes, en este caso espaciales, que establecen unos con otros: un gran cuerpo en movimiento (Bloom caminando = papalote volando) seguido de otros más pequeños dando saltos ondulantes (los vendedores de periódicos $=$ cola del papalote, haciendo piruetas en el aire [«capering,»]) y con extensiones láterales (los nudos de la cola del papalote $=$ los periódicos bajo el brazo). La intersección sémica queda 
constituida no por semas aislados sino por esta compleja configuración descriptiva.

En la metáfora del papalote, el grado construido aparece manifiesto casi en estructura de símil (Bloom seguido de los niños es como un papalote al viento). La segunda metáfora, en cambio, es mucho más abstracta, ya que se.da en el término ausente-barco/navegación-que hay que construir a partir tanto de la palabra «wake,» verdadero disparadero de esta segunda metáfora, como de la configuración descriptiva en la base de las dos metáforas: Bloom se aleja como un barco que al navegar deja una estela («wake») de espuma. La isotopía /marina/se activa entonces con "wake» ${ }^{19}$ cuyo primer significado como lexema nominal concreto es precisamente el de la estela que deja tras de sí un barco. Por otra parte, el sentido «estela de espuma» se ve reforzado por una blancura que en el nivel no metafórico de la descripción no parece ser pertinente: los periodiqueros difícilmente merecen tal calificativo en su desaliño; Bloom por su parte, lo sabemos, está vestido de negro y acaba de regresar de un funeral. Por lo tanto, «zigzagging white» no tiene sentido en el nivel denotativo de la descripción, aun tomando en cuenta la ambigüedad sintáctica que representa «the last.» Es difícil decidir si esta frase anafórica remite a Bloom o a los periodiqueros; ninguno de ellos, sin embargo, es susceptible de ser descrito en términos de blancura. Tampoco los periódicos parecen merecer tan albo calificativo. La metáfora del papalote tampoco parece incorporar esta blancura como parte constitutiva de su significación, incluso a pesar de la manera tan explícita con la que se califica a los nudos del papalote: «tail of white bowknots.» En cambio, en ese segundo nivel metafórico de significación, esta blancura zigzagueante puede sugerir la forma de la estela de espuma que deja un barco.

Así, en esta segunda metáfora, la misma configuración descriptiva es aparente a pesar de las variaciones en el nivel de la manifestación y del contenido de las descripciones (literal o metafóricas): un gran cuerpo (barco = Bloom) seguido por una estela blanca (los niños y la parte de blancura, aunque muy relativa, que tiene el papel del periódico = estela de espuma). El gozne que articula las dos metáforas sobre la base de una configuración descriptiva idéntica es la palabra "wake,» cuya compleja constitución semémica permite la activación de ambas metáforas de manera simultánea. En un primer momènto, el semema «wake» /a la zaga/, en conjunción con «breeze,» «kite» y «tail» activan la isotopía /aérea/ permitiendo la lectura de una primera metáfora; pero el semema "wake» /estela que deja un barco/, en conjunción con «breeze» $\mathrm{y}$ 《white»-Conectores pluri-isotópicos $-{ }^{20} \mathrm{y}$ con el tema general propuesto paratextualmente por el nombre del episodio «Eolo» (Ulises zarpando de la isla de Eolo con los vientos aprisionados en una bolsa), activa la lectura de la segunda metáfora: Bloom $=$ barco, $y$, debido a la significación 
simbólica articulada por la configuración descriptiva (como en el caso de «Hades»), Bloom = Ulises, el marino. Si bien el disparadero inicial de esta segunda metáfora es la polisemia de «wake»-cuyo primer significado, registrado por el Oxford Dictionary of English, es justamente «estela de espuma que deja un barco sobre la superficie del agua»_- ${ }^{21}$ la configuración descriptiva en la base del complejo de significación de esta metáfora establece también nexos intertextuales con el episodio correspondiente de La Odisea. Podemos entonces proyectar la triple incidencia de esta configuración descriptiva, en y a pesar de las múltiples variaciones que se observan en los contenidos de las dos metáforas y de la descripción en el plano denotativo. Así, podríamos abstraer el patrón de esa triple incidencia de la siguiente manera: un solo cuerpo en posición anterior (Bloom $=$ papalote $=$ barco), seguido por un rastro blanco.$($ los periodiqueros con los periódicos relativamente blancos bajo el brazo = la cola del papalote $=$ la estela de espuma de un barco).

El análisis de estos textos descriptivos nos permite concluir:

a) que la configuración descriptiva, lejos de ser una mera repetición de los detalles descriptivos en el nivel de la manifestación y en el de los objetos de la descripción, se presenta como un fenómeno relacional y de construcción de lectura; es la figura significante que traza la relación de ciertas particularidades descritas-independientemente del contenido específico de la descripción-lo que al reduplicarse en la descripción de otro objeto, ya sea similar o completamente diferente, cristaliza en figura y hace que las configuraciones percibidas entren en relación significante, con frecuencia de analogía;

b) que estas relaciones de analogía tienden a articularse de manera metafórica para reunir diferentes segmentos del relato y conferirles una dimensión de significado simbólico o ideológico-que cada uno de los segmentos aislados no contiene. Hemos visto cómo, de manera cumulativa, estas articulaciones metafóricas sobre la base de configuraciones descriptivas idénticas van dibujando un claro perfil ideológico en la narración de la vida de las prostitutas en la novela de Zola. $\mathrm{O}$ bien, las configuraciones descriptivas pueden constituir un puente intertextual que le confiera al texto dimensiones de significación míticas y simbólicas, como en el caso del Ulises, de James Joyce, al proyectarse de manera alusivo metafórica sobre La Odisea.

\section{Notas}

${ }^{1}$ Para un estudio más detallado de la descripción, no como pieza de ornamentación del discurso, sino como práctica textual con función y significación narrativas ver: Gérard Genette (1969), Philippe Hamon (1972; 1981), Luz Aurora Pimentel (1986; 1987).

${ }^{2}$ En este sentido nuestro concepto de configuración descriptiva es paralelo aunque define áreas completamente diferentes del concepto de configuraciones discursivas, 
definido por Greimas como: Les configurations discursives apparaissent comme des sortes de micro-récits ayant une organisation syntactico-sémantique autonome et susceptibles de s'intégrer dans des unités discursives plus larges, en y acquérant alors des significations fonctionnelles correspondant au dispositif d'ensemble (Greimas 1979, «configuration»).

${ }^{3}$ Emile Zola, Nana, (préface) Henri Mitterand. (Paris: Gallimard, «Folio,») 1977, 238. Todas las referencias subsecuentes al texto en francés serán a esta edición.

${ }^{4}$ Emile Zola, Naná. México: Porrúa, 1983 [1880], 122. Todas las referencias subsecuentes al texto en español serán a esta edición.

${ }^{5}$ Debo aclarar que los pasajes que he seleccionado para este trabajo analítico no son ciertamente los únicos en los que se observa la recurrencia y aun la evidencia de esta configuración descriptiva; espero, sin embargo, que sean suficientes en número y resonancia para ilustrar la pertinencia del modelo análítico que he propuesto.

6 James Joyce, Ulysses (New York: Random House, 1946), 98; 99. Todas las referencias subsecuentes al texto en inglés serán a esta edición.

7 James Joyce, Ulises (trad.) J. Salas Subirat (Buenos Aires: Rueda 1972, $6^{\mathrm{a}}$ ed.), 129-130. Todas las referencias subsecuentes al texto en español serán a esta edición.

${ }^{8}$ En ese sentido el traductor abusa al dar «tallados en piedra» por «hewn;》 la especificación «en piedra» es absolutamente impertinente, dados los propósitos de estas descripciones, como lo veremos en seguida.

9 «Apiñadas» («crowded»), «más frecuentes,» «apiñadas» («thronged»), «pasando» («streamed;» esta traducción, sin embargo, no toma en cuenta el sema /multiplici$\mathrm{dad} /$, central en la significación de "streaming»).

10 "Silenciosas,» «señalando,» «blanco silencioso,» «mudos.»

11 «Pesarosas,» «aflicción,» «prolongados gestos vanos en el aire.»

12 «extendiendo calmas manos,» «suplicantes,» «prolongados gestos vanos en el aire.»

${ }^{13}$ Para esta parte del análisis, y por las resonancias que la versión inglesa de $L a$ Odisea tiene en la obra de Joyce, he utilizado la extraordinaria traducción en verso de Richmond Lattimore: The Odyssey of Homer. (New York: Harper \& Row, 1965,1967). La versión española es de Luis Segala y Estalella: La Odisea. Prólogo de Manuel Alcalá (México: Porrúa, 1986).

${ }_{14}$ Para un modelo de narración metafórica ver Pimentel (1990, 34-67).

${ }^{15}$ El concepto de isotopía, originalmente formulado por Greimas en 1966 y afinado en 1979 (v. entrada «isotopie»), fue ampliado por Rastier (1972, 83-88).

${ }^{16}$ Cf. Grupo Mu $(1977,46 \mathrm{ss})$. Ver también el interesante estudio de Michel Leguern (1973) sobre la metáfora como fenómeno de ruptura isotópica.

${ }^{17}$ La intersección sémica, en la base de la significación metafórica, se construye con las unidades de significación semejantes aisladas de los dos campos semánticos distintos. (Cf. Grupo Mu 1977, 47). Ver también la entrada «metáfora» en Helena Beristáin (1985), y Pimentel (1990, 11-33).

18 «Barrilete» en la traducción de Salas Subirat.

19 Son muchos los significados de «wake,» entre los que podemos destacar «despertar» (como verbo); «vigilia,» «funeral,» «estela,» «rastro» (como sustantivo); «a la zaga» (como frase adverbial). 

(1975)

${ }^{20}$ Sobre la metáfora hilada y los conectores pluri-isotópicos, ver Phillipe Dubois

21 Wake: «the track left on the water's surface by a ship.» Oxford English Dictionary.

\section{Obras citadas}

Beristáin, Helena. 1985. Diccionario de retórica y poética. México: Porrúa.

Dubois, Phillipe. 1975. «La métaphore filée et le fonctionnement du texte.» Le Français Moderne 43 no. 3: 202-13.

Genette, Gérard. 1969. «Frontières du récit.» Figures I, Paris: Seuil.

Greimas, Julien Algirdas. 1976, 1966. Semántica estructural, Madrid: Gredos.

— \& J. Courtés, 1979. Sémiotique. Dictionnaire raisonné de la théorie du langage. Paris: Hachette.

Groupe $\mathrm{Mu}, 1982,1970$. Rhétorique générale, Paris: Seuil.

- 1977. Rhétorique de la poésie, Bruxelles: Complexe.

Hamon, Philippe. 1972. Poétique 2: 'Qu'est-ce qu'une description?' 465-85.

- 1981 . Introduction à l'analyse du descriptif, Paris: Hachette.

Leguern, Michel. 1973. Sémantique de la métaphore et de la métonymie. Paris: Larousse.

Pimentel, L. A. 1992. «La dimensión icónica de los elementos constitutivos de una descripción,» Morphé 6: 109-144.

1990. Metaphoric Narration: Paranarrative Dimensions in «A la recherche du temps perdu.» Toronto: $U$ of Toronto $P$.

— 1986 . «El espacio en el discurso narrativo: modos de proyección y de significación.») Morphé 1: enero-junio: 115-28.

—_. 1987. «Descripcíón y configuraciones descriptivas.» Acta poética 8: 41-68. 